\title{
Tracking online collaborative work as representational practice: Analysis and Tool
}

\author{
Johann Ari Larusson, Richard Alterman \\ Brandeis University, USA \\ johann@cs.brandeis.edu,alterman@cs.brandeis.edu
}

\begin{abstract}
In recent years wikis have enabled people to collaborate online as a community of practice by allowing content to be modified freely by any user. This platform has proven to be very successful despite its unconventional "anarchistic" and sometimes chaotic organization - Wikipedia being the most famous example. When people contribute to a wiki web site their work takes place within a representational system comprised of multiple distributed representations such as the wiki pages and external web sites related to the wiki topic. How much of the collaborative work involves transferring information between the different representations? How does the collaborative work emerge within the system and how is it used by the participants to accomplish their goal? We present WikiPlayer, a tool to visualize and replay the entire revision history of related wiki pages as they collectively evolve over time. The player allows us to track each user's contribution to a set of wiki pages, review the state of each page at any given moment in the history, and easily generate statistics helpful in analyzing the collaborative community of practice. The tool can be used to identify the collaborative work patterns that develop from the emergent interaction between the structure of wiki pages and the organization of the participants' representational work.
\end{abstract}

\section{Introduction}

Online communities of practice are social entities comprised of users who have overlapping or shared goals and interests. Technology that supports activity within an online community of practice takes several forms, ranging from alternate channels of communication, to the virtual meeting rooms, to wiki-based methods for sharing documents. The evaluation of 
the role of technology, and its design, in the productivity of online communities of practice is a significant and necessary step to engineering better environments for online collaboration.

Analysis of online interactions within a community of practice can be performed by tracking the representational work of the community; this style of analysis has been developed as part of the distributed cognition movement (Hutchins [4,5], Rogers [12], Norman [10]). Consider the case of a community of practice that collaborates vis-à-vis a wiki web site. The community creates representations within the wiki. Representations are transferred from other locations, such as external websites, email exchanges, or documents stored on a laptop, to the wiki site. The distribution of representational work and the organization of representational content on the wiki are significant features of how the community of practice carries out their collaborative work.

We have developed the WikiPlayer, a tool that enables researchers to identify collaborative work patterns within a community of practice as it emerges from their representational work. WikiPlayer enables the analyst to track the propagation of information between different representations, each user's contribution to a set of wiki pages, review the state of each page at any given moment in the history, and easily generate statistics helpful in analyzing the collaborative work of the community of practice.

In the study presented in this paper, WikiPlayer is used to explore the collaborative work within a community of users. The focus is on how the collaboration is managed by means of both the division of representational work and the emergent structure of the representational system that mediates the cooperative activity. Our results show that these factors can be tracked and are predictive of performance.

This kind of an analysis would not be possible without tools like WikiPlayer. WikiPlayer allows groupware researchers and designers to dig deep into the collaborative activities and study how the collaboration is carried out. The "over-the-shoulder" view of the collaboration gives a unique perspective of the online cooperative work, providing a detailed viewing of the organization and execution of the collaborative activity. The capability to better model the mechanics of online collaboration is a precursor to a more effective approach to designing technology to better support online communities of practice. 


\section{Background}

Collaboration is the process of interaction amongst people who share the same goal [2]. Examples of collaborations include non co-located employees of the same company who need to work together on a project or college students that are required to collaborate on class assignments. Often collaborators are faced with daunting tasks such as agreeing on a specific date and time where everyone can meet, sharing information between participants, and viewing each other's contribution. Development of online collaborative groupware aims at solving or simplifying these issues by supporting different place collaboration. One example of such a technology is a wiki invented by Ward Cunningham $[9,17]$ in 1995 . A wiki is a special kind of web site that supports asynchronous editing and has two distinguishing main features. First, every visitor to the site has the rights to edit everything, and editing is not discouraged but encouraged. Each member of the community of practice for a wiki site can freely edit both the content and the structure. Second, most wikis make public all past revisions of the articles that the users create, providing them as well as researchers with a rich record of the collaborative activity.

We have introduced wikis for several educational and research related tasks. We have used wikis as a research platform to collect data of online collaborative activities to support our studies of synchronous and asynchronous collaboration. In the spring of 2006 one of the assignments in the Internet \& Society course taught at Brandeis University required the class to collaboratively write a paper on one of the books from the reading list using a wiki as a collaborative platform. In another class, Computational Cognitive Science, wikis were used to collect replayable data of online collaboration both to support lectures and to use as a source of data for student term projects. In a Human-Computer Interaction (HCI) class, a wiki platform provided a repository for exchanging ideas and sharing material within a team of students engaged in a group term project. For each of these wiki-based tasks we have developed several techniques for reviewing transcripts of the online community of practice.

Our lab has developed several groupware applications that produce complete replayable transcripts of online user behavior [6]. A complete transcript, of the sort produced by these applications, enables the analyst to replay all online activity from an "over-the-shoulder" perspective as if he or she were viewing a video. Another vehicle of analysis we have developed extracts the discourse from a transcript, displaying the conversational part of the collaboration as hierarchically organized text interspersed with one-line summaries of web activity and wiki page edits. In this paper we 
present the WikiPlayer which is another example of an analysis tool. Using the WikiPlayer, an analyst can closely examine the collaborative work patterns within a community of practice as they emerge as a product of their representational work on a wiki.

As a research tool, the WikiPlayer facilitates researchers in identifying collaborative work patterns and track the online collaborative activity and how it is affected by the representational system. The WikiPlayer enables close examination into how participants organize their representations, the propagation of representations between alternate forms, and the division of representational work.

WikiPlayer also has value in an educational context. Teachers can easily evaluate individual student contributions in a collaborative wiki assignment such as the one described above. The WikiPlayer can be used by students to better understand and keep track of each other's work during class projects as well as providing them with an in-depth view into the activities when studying analysis, design, and cognitive engineering methods that apply to online collaboration.

In this paper, we will look at the WikiPlayer as a tool for conducting research of online collaborative activities. Using the WikiPlayer we investigated how collaboration on wiki sites is carried out with respect to the representational system. Since wikis make available the entire revision history of a collaborative wiki project the data set can be enormous. Data mining on such a vast set of information is not a straightforward task, let alone trying to analyze the data in terms of the entire representational system - the WikiPlayer addresses these issues.

The data we analyzed was collected from an experiment where student were engaged in a collaborative advising task that was mediated by a wiki. The wiki used for the experiment was MoinMoin [16] one of many clones of the original wiki developed by Ward Cunningham. MoinMoin shares some of the main features with other wiki clones such as allowing all users to edit the content anonymously, and providing the revision history of the wiki pages.

Our main results show that there is a close relationship between the success of the collaborative project and the amount and kind of representational work carried out by the participants. The success of the activity is dependent upon how the participants recognize, share, and manage representations vis-à-vis the emergent representational system. We used the WikiPlayer to identify the kinds of representations and work that each team of users performed. Our results show that how a group organizes their representational work is predictive of how well they achieve their cooperative aim. In itself this result is not surprising. What is of value is that 
we can use the WikiPlayer to construct detailed models of how each group works together on their collaborative representational task.

\section{Wiki technology}

A wiki is a freely expandable collection of interlinked web pages. In essence it is a database of documents that can be easily edited by anyone using an editor accessible through most web browsers [9]. People collaboratively write documents in a very simple markup language where they can either edit an already existing page or create new pages. Wiki pages, as presented to each visitor upon his or her first visit to the wiki site, are more or less just plain HyperText Markup Language (HTML) pages. The contributing user never edits the HTML code. Instead, using a simple editor the user edits the source of the wiki page, often referred to as the wikitext, which can also be augmented with a simplified markup language to indicate various structural and visual conventions [15]: wikis provide a simplified process of creating HTML pages. To contribute to a wiki topic, users simply click on the "edit" button of the wiki page they wish to edit and the page will display the editable wikitext inside an editor.

\section{Revision history: Tracking page edits}

Most wikis, such as MoinMoin, include various tools that focus on providing users with a simple way of monitoring the state of the wiki as it changes. One such feature is the archival system. Wikis record and log every change made to a page no matter how insignificant the change might be. An important part of online collaboration on a wiki is keeping track of edits made to a page. The most recent changes to the wiki are accessible by clicking on a "Recent Changes" link that will open up a page that lists all the recent changes in a simple manner. This functionality helps users keep track of the evolution of the topic they are interested in and monitor the contributions of their fellow collaborators.

One of the features provided by MoinMoin and most other wikis is the availability of raw data. Wikis make accessible the entire revision history of all documents created on its servers, a valuable source of information for researchers analyzing online collaborative activities. The archive can encompass tens, hundreds, or even thousands of entries containing the actual revision and meta information. 


\section{Analyzing the revision history}

In the previous literature researchers have studied collaboration via wiki by examining the page revision history. Viegas et al focused on visualizing the revision history of a single page to identify relationships between multiple document versions in order to reveal patterns within the wiki context that could be useful in other collaborative situations as well [14]. However their methods do not take into consideration the evolution of the collaboration in terms of the representational system. Hutchins has produced detailed models of the representational systems for both the airplane cockpit and the bridge of a navy vessel $[4,5]$. When collaboration occurs within a representational system, work patterns emerge as a result of the representational work required on behalf of the participants to carry out and complete their task [7].

A wiki project can contain multiple related wiki pages on a specific topic where each page spans a specific subsection of the topic. This organization is frequently observed on sites such as Wikipedia. The wiki project in addition to other external representations such as remote web pages used as information sources create the representational system. The collaboration occurs within this system and the wiki revision history describes the process of the collaborative activity. Therefore, when analyzing online collaboration it is important to look at the emergence of the collaborative wiki project as a whole and not just the evolution of a single page. The revision history archive can be quite large, even for only few pages. As an example, the data we collected included roughly 2450 files, describing about 500 unique revisions to the wiki pages and totaling 2.4MB of raw text. Making sense of the history of the pages that the data set describes is challenging and analyzing the relationship between the pages at the same time is not straightforward.

\section{WikiPlayer}

The MoinMoin wiki provides an option of viewing the changes that have been made recently enabling users to easily keep track of the constantly updating wiki, and see what documents were recently changed, who changed them, and when. However, there are two problems with the design of this interface. First, it is not possible to easily observe the evolution of related pages at the same time. Second, this only facilitates observation of recent changes and not the entire history. The history flow visualization technique [14] addresses one of these issues but has one major drawback, 
it only enables researchers to easily track trends in the collaboration by viewing the revision history a single page at a time. This does not take into consideration the importance of viewing the collaborative work effort by the community in terms of the representational system and how it affects the activity.

As discussed above, the WikiPlayer has several significant functionalities. The focus of the paper will be on how the WikiPlayer can be used to simultaneously replay multiple wiki pages in a manner that makes it easier to identify patterns of representational work that emerge within a group.

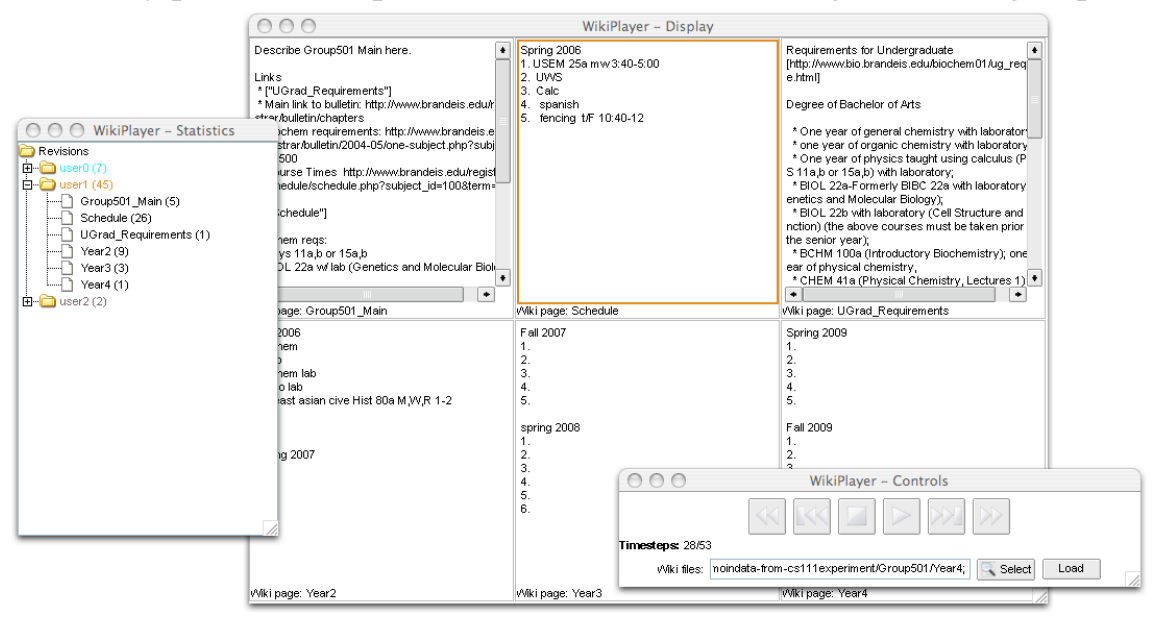

Fig. 1. The WikiPlayer user interface

\section{User interface}

The WikiPlayer was written in Java and thus can operate on all platforms that can run the Java Runtime Environment (JRE). The base application is divided into three independent components (also shown in the figure above):

- WikiPlayerDisplay - Displays/visualizes the wiki revision history.

- WikiPlayerConsole - Controls to e.g. play, stop, fastforward the history.

- WikiPlayerStatistics - Display statistics extracted from the history.

WikiPlayerConsole is the window that contains menu options to load the history into the player as well as "VCR style" controls that can be used to move around the revision history just like if one would be playing his or her favorite videotape. Once the history has been loaded into the WikiPlayer it is possible to play it forward/backwards, stop, step forward 
or backwards, and fast forward/backwards. The goal is to make the interaction with the revision history as simple and intuitive as possible.

The design of the WikiPlayer enables compatibility with all types of wikis. The WikiPlayerConsole asks a LoaderManager to parse the revision history, that a user requested, on its behalf. The manager uses auxiliary "loaders" (Java classes) written specifically to parse a history from a certain wiki type. Following a special architecture each loader returns the history in a structured format, uniform for all wikis, that the manager understands and can use to prepare the history data for playback. The preparation involves for example synchronizing histories of individual pages and assigning each user with a pseudonym and a unique color. Currently WikiPlayer supports MoinMoin wiki and MediaWiki (the wiki used on Wikipedia).

The WikiPlayerStatistics component receives the prepared history from the manager. It examines the entire revision history and generates useful statistics such as the total number of edits per page per each individual user. The significance of each contribution can be measured by comparing the size/length of each revision to the overall content length of the wiki page. This enables analysts to distinguish between users that made a lot of edits with little content and users that made few or many edits but in either case contributed significantly to the content of the wiki. The statistics are distributed onto each individual user and presented using their pseudonym and color in the WikiPlayerStatistics window.

Once a revision history has been loaded the controller notifies the WikiPlayerDisplay window so it can initialize itself. The display is divided into subsections similar to a checkerboard each square representing a wiki page. Different groups construct different numbers of wiki pages, so the size of the checkerboard for a given group will vary accordingly. Upon starting the replay each contribution made by a user to a page is easily tracked by highlighting his or her changes to a page using the assigned color. This facilitates identifying the contribution and keeping track of his work on the wiki project when he moves about the different pages as the group collaborates. During playback the revision made to a document is visualized on its respective document item on the display window and all other documents that were not modified at this timestep retain their appearance from the previous timestep.

\section{Extending the WikiPlayer}

To enhance the capabilities of the WikiPlayer it is possible to write plugins that add a specific set of features or services to the player. The plugins can 
be seamlessly integrated using access points and methods provided by the WikiPlayer Plugin Application Program Interface (API). A few plugins have already been developed for the tool. CedarChatParser, was written to extract the chat dialogue from the transcripts produced by one of our groupware applications and align the discourse with the wiki revision history so one could monitor the communication between group members taking place "off the wiki" parallel to their work "on the wiki" as they constructed the representational system.

\section{Experiment: Data on online collaboration}

In the fall of 2005, a course in Computational Cognitive Science was taught at the Department of Computer Science at Brandeis University. There were 28 students; a mix of graduates and undergraduates as well as computer science majors and non-majors. The course focused on introducing students to theoretical material on online cooperation and joint sensemaking [1].

The class was divided into teams of 2 to 5 students. The experimental task was organized in such a way that it would encourage online sensemaking and collaboration. The experimental platform used was CEDAR, an application wrapper around a wiki web site that provides a few additional collaborative tools and enables replay of the collaborative wiki assignment at the user interface level [1]. The goal was to construct a collaborative task where there were multiple dependencies among the various subtasks. Each team acted as an academic advisor that was required to develop at least a two-year schedule of classes for an incoming freshman. Part of the task was to post the student's schedule for each year in chronological order within a certain amount of time.

The groups collected information from sources on the World Wide Web including course listings and requirements from the University Registrars office. They combined this information with their own experiences and preferences developed throughout the collaborative activity and constructed the class schedule in the form of a wiki page, using MoinMoin wiki. Halfway through the session, the teams were interrupted and informed that their freshman advisee had decided to add a specific minor to his or her schedule. When they were finished, the teams were required to submit the schedule to "the student". The student critiqued the schedule, and always found some changes that needed to be made - usually this meant a change in course selection or sequence of courses - forcing the teams to rework the schedule. This required the teams to collaboratively 
revisit older parts of the schedule and modify them while continuing to work on planning the unfinished semesters.

\section{Representational work}

CEDAR was developed using our homegrown groupware engineering toolkits THYME and Sage [6]. CEDAR automatically produces replayable transcripts of the online practice. A VCR-like replay device, provided by CEDAR, allows the analyst to review all the online activity of the subjects at the user interface level, searching for domain meaningful events like a chat event or a web browser event. More detail on the development and usage of CEDAR are provided in [8]. One disadvantage of the replay device in CEDAR is that reviewing the representational work that the students do on the wikis is cumbersome.

We used the WikiPlayer to replay the revision history of the wiki that the students used in the academic advisor assignment; in total we replayed the wiki history of 11 student teams. We used the distributed cognition (DCOG) framework as a basis for analyzing the transcripts. The idea of distributed cognition is that cognition is embedded into a large system of internal and external distributed representations (Hutchins [4,5], Rogers [12]). Within the DCOG framework, cognition can be tracked as the propagation of representational state within a functioning system of actors, representations, and cognitive artifacts [3]. Thus, when people collaborate online using a wiki their collaboration is mediated by the wiki in terms of the representations available to the collaborators. Each individual has their own interpretation of the activity and of the content stored on external representations, such as books or remote websites, which contain information relevant to their collaborative project. The wiki pages that the collaborators create and maintain are significant components of the representational system in which the collaborators work. It is for these reasons, that an analysis of cognitive performance (the collaboration on the wiki) can be framed in terms of the entire representational system in which the behavior is embedded $[4,5]$.

Our observations revealed some fascinating representational work patterns employed throughout the collaborative activity. We investigated four interesting representational patterns in more detail. These patterns represent the techniques that the groups developed during the collaboration to deal with the hindrances caused by the nature of the task, information used, and (lack of) support for representational work. The teams often collaborated on representational activities to deal with interruptions to the 
work process like the introduction of a minor for the freshman. Each pattern of representational work can be characterized by a distribution and division of labor, the management of the collaborative task, and the organization of representational content. We will now describe in more detail the main representational activities that we observed.

\section{Representational information transfer (mapping)}

To successfully finish the class schedule the teams had to locate information on the web from several sources, including the University Registrars' Office and the web pages for various departments. An effective strategy was to transfer information from a web page to a wiki page, while perhaps adding some additional organizational structure. This strategy was effective because it made the information more readily available for continued access.

\section{Representational task management}

In some cases, we observed groups creating checklists on a wiki page. For example, the checklists were used to prevent course duplications and to manage group effort. The subjects sometimes used information copied from web pages on to wiki pages as a checklist. Thus, we see the propagation of representation as it is transformed and modified to meet the demands of alternate tasks: first as a statement of requirement, then organized to make repeated access more efficient, and finally as a "checklist" that would insure the completion of all elements of the task.

Students annotated the checklists using words such as "DONE", "FINISHED", or marked entries with asterisks to keep track of the activity. On some occasions they even used links to the wiki pages as markings indicating that a certain requirement had been fulfilled in the schedule. The link would then take the user to the wiki page were the requirements were satisfied.

\section{Representational task organization}

Some groups decided to employ a "divide-and-conquer" strategy to finish the assignment. Each group member was assigned a specific task that he or she only worked on during the entire assignment. Up to a point this strategy is effective. The problem is that there are dependencies, for example, between requirements, and consequently too much division of labor can 
potentially produce scheduling conflicts that lead to excessive repair work. Thus, many teams avoided explicit "divide and conquer" strategies, preferring to collaborate in detail on the majority, if not all, of the wiki pages. Examples of this kind of fine grained collaboration over representational work include: copyedits of each other's work, multiple subjects adding information to single wiki pages, and the collaborative reorganization of material that was copied from a web page to a wiki page. This kind of anarchic approach to representational work is consistent with the philosophical approach of sites like Wikipedia.

Whether or not the subjects deploy a "divide-and-conquer" strategy is one important determinant of the overall organization of the representational work within a team of subjects. The kinds of representations the subjects worked with, both number and kinds of wiki pages, is another significant determinant of how the collective representational work is organized. How a group works out the details for each one of these organizational tasks largely defines the flow of representations as the group performs their task.

\section{Organization of the representational system}

We used the WikiPlayer to identify different kinds of organizational structures for the collection of wiki pages produced by a single group. The teams mainly used two different structures to organize their wiki pages. Some groups only had a few pages, and few if any links between them resulting in a very "flat" and "horizontal" structure of independent pages not easy to navigate. Other teams divided information into many pages (representations) adding a hierarchical organization to the structure of the wiki, making it easier to navigate, with relevant information grouped together at the same or close locations on the wiki. The latter organizational scheme often included a main overview page linking to relevant sub pages and/or related pages.

\section{A quantitative measure}

Our analysis focused on the four representational work patterns described in the previous section.

For each work pattern we assigned points on the scale of 0 to 10.0 was the lowest amount of effort put into a representational activity by a team and 10 indicated the highest amount of effort. The points were assigned differently to each pattern and the grading strategy is explained in Table 1. 
Table 1. Representational work pattern grading policy.

\begin{tabular}{|c|c|c|c|}
\hline & Pattern title & Description & Grading policy \\
\hline 1 & $\begin{array}{l}\text { Rep. information } \\
\text { transfer }\end{array}$ & $\begin{array}{l}\text { Subjects transfer in- } \\
\text { formation from web } \\
\text { pages to Wiki }\end{array}$ & $\begin{array}{l}1 \text { pt. assigned for trying } \\
3 \text { pts. for each requirement trans- } \\
\text { ferred } \\
-1 \text { pt. for each requirement section } \\
\text { sharing a page. }\end{array}$ \\
\hline 2 & $\begin{array}{l}\text { Rep. Task man- } \\
\text { agement }\end{array}$ & $\begin{array}{l}\text { Track progress using } \\
\text { checklists }\end{array}$ & $\begin{array}{l}0 \text { pts. for no lists. } \\
5 \text { pts. for lists sharing a wiki page. } \\
10 \text { pts. for detailed list on a separate } \\
\text { page. }\end{array}$ \\
\hline 3 & $\begin{array}{l}\text { Rep. task organiza- } \\
\text { tion (collaboration) }\end{array}$ & $\begin{array}{l}\text {-Multiple subjects edit } \\
\text { each Wiki page. }\end{array}$ & $\begin{array}{l}\text { For each team, the percentage of } \\
\text { wiki pages where two or more par- } \\
\text { ticipants collaboratively edited the } \\
\text { page. }\end{array}$ \\
\hline 4 & $\begin{array}{l}\text { Rep. system orga- } \\
\text { nization (wiki) }\end{array}$ & $\begin{array}{l}\text { Hierarchical structure } \\
\text { of Wiki }\end{array}$ & $\begin{array}{l}0 \text { pts. for no hierarchical structure. } \\
5 \text { pts. for using some form of hierar- } \\
\text { chical structure sharing a wiki page. } \\
10 \text { pts. for using well organized } \\
\text { structure on a separate page. }\end{array}$ \\
\hline
\end{tabular}

To measure the total amount of representational work for each team we calculated a score, on a scale of 0 to 100 , by summing the scores assigned to each representational work pattern that a team performed. We decided that all of the representational work patterns were equally important - each weighing $25 \%$ of the total score (denoted by $w=2.5$ ). The formula to calculate the total amount of representational work is shown below.

$$
\sum_{i=0}^{t} w P_{i} \quad \begin{aligned}
& \text { Where } t=4 \text { (total no. of patterns) } \\
& \text { For an integer } 0 \leq i \leq t
\end{aligned}
$$

To explain the evaluation we will use one group as an example. One successful group transferred all of the requirements earning 10 points for the first pattern, however major and minor requirements shared a single wiki page, which means that two points were subtracted resulting in a total score of 8 for pattern $P_{1}$. Using the copied information they created a new separate wiki page for the sole purpose of being used as a checklist to manage the ongoing activity earning them another 10 points for pattern $\mathrm{P}_{2}$. This group created in total 10 wiki pages and two or more team members collaboratively added content to 6 pages (or $60 \%$ ) thus the overall score for pattern $\mathrm{P}_{3}$ is 6 (since all pattern grades are on a scale of 0 to 10). This group put great effort into organizing the wiki into a good hierarchical structure including an overview link page into the structure that earned them 10 points for pattern $\mathrm{P}_{4}$. Since all the patterns constitute representa- 
tional work we calculate the final amount of representational work done by the group by summing the scores for the individual patterns. Since the final score is on the scale of 0 to 100 we need to multiply each individual score by the weight factor $w$. We calculate the final score according to our formula above:

$2.5 \mathrm{P}_{1}+2.5 \mathrm{P}_{2}+2.5 \mathrm{P}_{3}+2.5 \mathrm{P}_{4}$ therefore

$2.5 * 8+2.5 * 10+2.5 * 6+2.5 * 10=85$

Thus, the total amount of representational work that the group did during the assignment was 85 on the scale of 0 to 100 .

\section{Results}

Analyzing a revision history for a wiki is helpful to gain insight into how online collaboration is performed. WikiPlayer proved to be a useful analysis tool to identify collaborative work patterns and how they are influenced by representational activities carried out by the teams within the representational system. We will now present some statistical corroboration to support our observations.

6 of the 11 student teams successfully finished the assignment - producing a complete and detailed class schedule for the incoming freshman. Those teams that finished tended to be more creative at using the wiki in various ways to manage their representational task [Figure 2].

\section{Groups that finished}

The 6 teams that finished were more effective at converting both their collaborative task and collaborative meta-task into representational work [Figure 2]. As a result, their representational system was richer and more effective. There was one exception to this rule. The outlier team included only two students, thus the coordination of their effort was less difficult than that of the other groups that finished the task, who had 3 to 5 team members and consequently more complicated coordination problems. In other words, the structure and organization of the flow of representations within a team is, not surprisingly, dependent on the size of the team. As the size of the team varies, the kinds of coordination problems change, and consequently alternate schemes for the representational task become more effective. With only two team members, there are fewer coordination problems, and thus the introduction of representational work to manage the coordination of the activity is not necessarily required. 
All the successful groups did significant amounts of copying from external websites to the wiki. A review of the data using the WikiPlayer showed the successful groups selectively copying only relevant and useful material. These teams also put effort into re-organizing the information once it was copied to a wiki page to better support the use of the information. For example, copied information that was subsequently used for a checklist, was modified by structuring the information to make it easier to annotate with checkmarks.

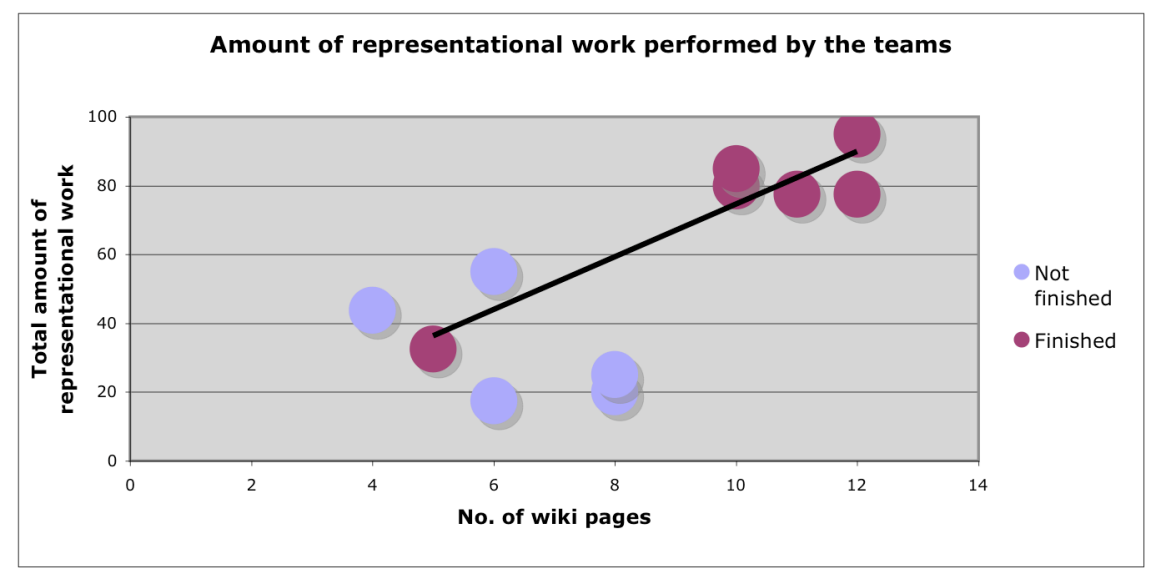

Fig. 2. Total amount of representational work.

All of the successful groups except for the outlier relied on using representations to keep track of the advancement of the task, using a wiki page as a checklist. For example, one group used the wiki page describing general university requirements, that they transformed from an external website, as a checklist with a mark of " $\mathrm{x}$ " as an indication that the requirement had been met in the schedule.

The successful teams collaborated on average on at least $50 \%$ of all the wiki pages that they created. About half of those teams collaborated on no less than $70 \%$ of their wiki page collection. Overall the level of collaboration amongst the successful teams was much higher than the teams that did not finish; this is understandable since two of the unsuccessful teams did not collaborate on a single page.

The majority (83\%) of the successful teams also spent a lot of their collaborative work organizing and structuring the representational system including the information mapped from external representations. Dividing up the content and grouping related information together on different locations on the wiki and linking them together forming a hierarchical repre- 
sentational structure proved to be very valuable to the teams as this enabled team members to quickly locate relevant information.

\section{Ineffective groups}

One consistent failing of the ineffective groups is that they did not use the wiki as a caching device for collecting information from web pages. More than $50 \%$ of the unsuccessful teams did little or no transfer of information (mapping) between representations whether it was between internal wiki pages or from external sources to the wiki as shown in Figure 3. Those that tried did very minimal copying often transferring non-useful information or not enough.

Further review of the data using the WikiPlayer also shows that the unsuccessful teams did not create checklists to manage their collective work. Groups without checklists often had individuals repeat work that had already be done by another team member.

By reviewing the data through the WikiPlayer we observed that a "divide-and-conquer" strategy was not optimal. It did reduce the representational work for the group, but at a high cost. Because of the many dependencies that exist between required courses and the scheduling conflicts between courses, the "divide-and-conquer" strategy produced numerous scheduling problems that required enormous amounts of work to fix.

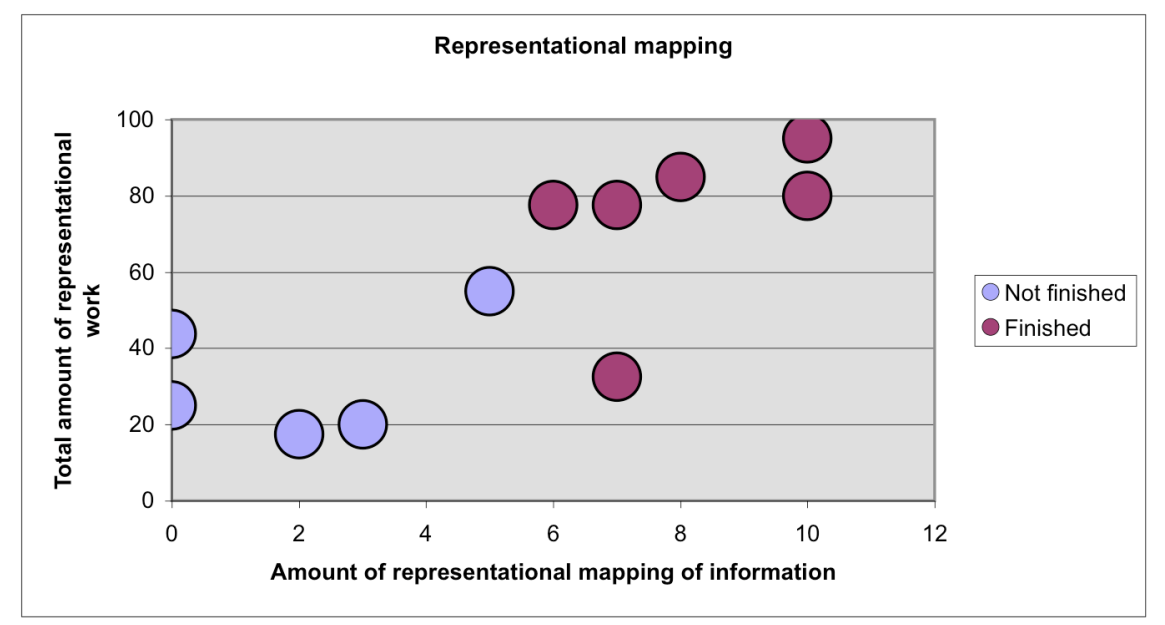

Fig. 3. Total amount of representational mapping of information. 
About half of the unsuccessful teams did not organize the wiki pages into a hierarchical structure. This was also a suboptimal strategy. When the ineffective groups introduced hierarchical structure it was not to organize the wiki pages, rather it was embedded into the content of some wiki page, thus reducing the impact on the flow of information within the representational system.

\section{Hierarchical clustering}

We wanted to see if other analysis tools would corroborate our observations of the representational work patterns. Using hierarchical clustering methods to group the data together we are able to visualize trends in the collaboration.

We used the Hierarchical Clustering Explorer (HCE) developed at the Human-Computer Interaction Lab at the University of Maryland [12]. This tool can be used to analyze multidimensional data sets such as the statistics we extracted from the wiki revision history using the WikiPlayer.

The software applies a hierarchical clustering algorithm without a predetermined number of clusters, and then enables users to determine the natural grouping with interactive visual feedback. To use the tool we transcribed by hand the statistics obtained from the WikiPlayer and from a brief "by-hand" analysis onto a tab-delimited format that could be loaded into the tool. HCE groups teams together based on their score on different evaluation parameters.

Using the Hierarchical Clustering Explorer (HCE) we were able to identify similarities between the teams based to the evaluation parameters they received from our analysis on representational work. The parameters included the following:

1. Did the group finish the assignment?

2. Team collaboration on wiki pages.

3. Maximum representational mapping by the team.

4. Minimum representational mapping by the team.

5. Hierarchical structure of the representational system.

6 . Was the class schedule divided onto semesters per page?

7. Was the class schedule divided onto years per page?

As we expected, the results from HCE supported our observations. HCE presented the results graphically where all the teams were automatically divided into two separate groups, one that included teams that finished the assignment and one that included unsuccessful teams. The clustering shows that there is a clear connection between the success of the assign- 
ment and how the teams organized their representations, the propagation of representations between alternate forms, and the division of representational work. The teams that were able to convert most of the collaborative activity into representational work all finished the assignment.

\section{Future work}

We believe that the WikiPlayer can evolve into a powerful analysis tool for online collaborative activities mediated by a wiki web site. We plan to automate many of the analyses we currently do by hand and explore alternate methods for visualizing the flow of representations.

The plugin API has already been used to extend the capabilities of the WikiPlayer. CedarChatParser that was described earlier is an example of a plugin. RepTracker, which is currently under development, is another example. RepTracker tracks the propagation of representation within the wiki; it uses Latent Semantic Analysis [10] to calculate the similarity between two wiki pages or two versions of the same page. The output of RepTracker will be a visualization of the flow of information between representational forms.

\section{Conclusions}

Online communities of practice consisting of users that share the same goals, tasks, and interests need technology that supports their work. A significant step towards engineering better environments for online collaboration is to evaluate its design and analyze the role of the technology in terms of the productivity of the online community.

By analyzing the community of practice and the online interaction in terms of the representational system we can track the representational work of the community. The distribution of representational work and the organization of representational content are significant features of how the community of practice carries out their collaborative work.

In this paper we discussed an approach to evaluation and a tool that enables it. The WikiPlayer simultaneously replays the entire state of the representational system by aligning the individual revision histories for each page revealing the shape of the entire representational system at each timestep, and how it evolves through time as a result of the representational activities. The way the WikiPlayer replays and visualizes the revision history of the representational system allows one to closely examine 
how the groups carried out these representational activities. Without the WikiPlayer the examination of the large amounts of data is a truly daunting task.

Using the WikiPlayer we were able to replay the entire revision history of a collaborative wiki project and identify four different representational work patterns. The results of our analysis showed a significant relationship between how each team organized their representational system and work, and how effective they were at achieving their collaborative aim.

Building analysis tools such as the WikiPlayer allows groupware researchers and designers to dig deep into the collaborative activities and study how the collaboration is carried out. The "over-the-shoulder" view of the collaboration gives a unique perspective of the online cooperative work within a community, tracking by means of each member's representational activities. The capability to better model the mechanics of online collaboration is a precursor to a more effective approach to designing online representational systems.

\section{References}

1. Alterman, R. The Impact of "Reflective HCI" on teaching in the Classroom. CHI 2006 Workshop on "Reflective HCI". April 2006.

2. Clark, H. H., Using language. Cambridge: Cambridge University Press, 1996.

3. Hutchins, E. and Klausen, T. Distributed cognition in an airline cockpit. In Engeström, Y., Middleton, D. (Eds.), In Communication and Cognition at work. Cambridge University Press, Cambridge. 15-34, 1992.

4. Hutchins, E. (1995a) How a cockpit remembers its speed. Cognitive Science 19(3) $265-288$.

5. Hutchins, E. (1995b) Cognition in the wild. Cambridge, MA: MIT Press.

6. Landsman, S. A Software Lifecycle for Building Groupware Applications: Building Groupware On THYME. PhD thesis, Brandeis University, 2005.

7. Larusson, J.A., Tulinius, V.O., \& Alterman, R. Measuring the representational work of pilots during an approach briefing. Technical Report CS-05-261. Available at http://www.cs.brandeis.edu/ johann (2005). 
8. Larusson, J.A., Alterman, R. Integrating collaborative technology into the interdisciplinary classroom. Technical Report CS-06-262. Available at http://www.cs.brandeis.edu/ johann (2006).

9. Leuf, B., Cunningham, W. The Wiki Way. Adison-Wesley, 2001.

10.Norman, D.A. (1993) Things that makes us smart. Perseus Books, Cambridge, MA.

11.Purandare, A., Pedersen, T. SenseClusters - Finding Clusters that Represent Word Senses (Purandare and Pedersen) - In Proceedings of the Nineteenth National Conference on Artificial Intelligence (AAAI04), July 25-29, 2004, San Jose, CA (Intelligent Systems Demonstration)

12.Rogers, Y. \& Ellis, J. (1994) Distributed cognition: an alternative framework for analyzing and explaining collaborative working. Journal of Information Technology 9, 119-128.

13.Seo, J. Information Visualization Design for Multidimensional Data: Integrating the Rank-By-Feature Framework with Hierarchical Clustering. PhD thesis, University of Maryland, 2005.

14.Viegas, F. B., Wattenberg, M., \& Dave, K. Studying Cooperation and Conflict between Authors with History Flow Visualizations. In Proc. CHI 2004, ACM Press (2004), 575-582.

15.Article on Wikipedia: http://en.wikipedia.org/wiki/Wiki

16.MoinMoin wiki site: http://moinmoin.wikiwikiweb.de/

17.Wiki Wiki Web http://c2.com/cgi/wiki?WikiWikiWeb. 